\title{
Reseña del XLVI Congreso Anual Colegio Mexicano de Medicina Crítica
}

Highlights from the 46th COMMEC Annual Congress

Revisão do XLVI Congresso Anual Colegio Mexicano de Terapia Intensiva

José de Jesús Zaragoza Galván*

Apreciable Editor, miembros del Colegio Mexicano de Medicina Crítica, público en general:

EI XLVI Congreso Anual del Colegio Mexicano de Medicina Crítica (COMMEC), celebrado a finales del mes de octubre 2019 en la ciudad de León, Guanajuato, se caracterizó por fomentar la integración nacional de los médicos especialistas en medicina crítica y especialidades afines. De hecho, el lema del congreso fue «fortaleciendo el trabajo en equipo»; nuevamente ha quedado de manifiesto la calidad y excelencia de la medicina crítica mexicana.

Este Congreso en particular fue estimulado desde el eje central de nuestra asociación: los capítulos (https://commec.org/capitulos/). Ellos son grupos de profesionales dedicados a la atención del enfermo en estado crítico que comparten interés en un área específica de la especialidad y que se agrupan con el propósito de trabajar conjuntamente en los campos de la investigación, docencia y enseñanza. Ellos mantienen la calidad y constituyen la parte esencial del COMMEC.

La investigación en medicina crítica en México ha crecido exponencialmente en calidad y cantidad. La investigación de alta calidad metodológica, estadística y ética es el camino a la excelencia de nuestra asociación. Este año, se recibieron 91 trabajos libres en resumen presentados en cartel y 23 artículos a texto completo participantes para el «Premio Dr. Mario Shapiro». El trabajo de investigación mejor calificado fue el titulado: Aclaramiento del déficit de base estándar como pronóstico de mortalidad en choque séptico del Dr. Gabriel García Gómez y colaboradores del Hospital de Especialidades No. 14 CMN «Adolfo Ruiz Cortines», del Instituto Mexicano del Seguro Social (IMSS) de Veracruz. El segundo lugar otorgado al reporte de Trombosis venosa cerebral en la Unidad de Cuidados Intensivos Neurológicos del Instituto Nacional de Neurología y Neurocirugía del Dr. Uriel García Cabrera del Hospital CMN Bajío UMAE No. 1 de León, Guanajuato, también del IMSS; y en tercer lugar el Dr. Eduardo Alberto González Escudero con el trabajo Factores asociados a

\footnotetext{
* Colegio Mexicano de Medicina Crítica.

Este artículo puede ser consultado en versión completa en www.medigraphic.com/medicinacritica
}

mortalidad en el adulto mayor con choque séptico del Hospital de Especialidades No. 14 CMN «Adolfo Ruiz Cortines", del IMSS de Veracruz.

En esta ocasión recibimos a más de 1,000 asistentes, de los cuales 820 fueron congresistas provenientes de toda la República. Participaron más de 140 profesores nacionales de 22 estados y 31 extranjeros de diferentes países: Argentina, Bélgica, Colombia, Ecuador, España, Estados Unidos, Francia, Italia y Panamá. La industria tuvo una participación sobresaliente con 32 stands y el patrocinio, asistentes, profesores, talleres y simposios durante todo el Congreso.

Por tercer año consecutivo, la jornada diaria iniciaba con la presentación de casos clínicos reales y con buena asistencia que permite el intercambio de ideas y aprendizaje de todos. Habíamos iniciado actividades el lunes 28 y martes 29 de octubre con 13 cursos precongreso. Todos ellos con temas de interés actual comparables en calidad con los de cualquier congreso de la especialidad de medicina crítica a nivel mundial: monitoreo hemodinámico, USLS Winfocus, taller de donación de órganos de la Federación Panamericana e lbérica de Medicina Crítica y Terapia Intensiva (FEPIMCTI), sepsis, ventilación mecánica, entre otros.

La ceremonia de inauguración incluyó dos conferencias magistrales de muy elevado nivel académico impartidas por la Dra. Hetherlee Bailey, presidente de la Society of Critical Care Medicine (SCCM): «The Continuum of Care: It's not a puzzle it's a cycle»; y el Dr. Andrés Esteban: «Evolución de la ventilación mecánica en México». De manera anual, el COMMEC reconoce la trayectoria de un intensivista y sus aportaciones a la medicina crítica nacional. Este año, tuvimos el honor de corresponder los logros del Dr. José J. Elizalde González, presentado por el Dr. Juvenal Franco.

Siguiendo con el programa inaugural, el Dr. Ulises Cerón Díaz dirigió la ceremonia de celebración del 40 aniversario de la fundación de la FEPIMCTI. Se entregó una placa conmemorativa al COMMEC como sociedad anfitriona de la fundación, y al Dr. Andrés Esteban un reconocimiento como firmante original del acta.

Después del mensaje de la Dra. Nadia Barroso, Presidente del Colegio de Medicina Crítica y Terapia Intensiva de Guanajuato, el Dr. Moisés Andrade Quezada Coordinador General de Salud Pública del estado de Guanajuato, en representación del gobernador Lic. Diego 
Sinuhué Rodríguez Vallejo, dirigió el mensaje inaugural del congreso.

Cuatro días de intensa actividad académica para médicos y enfermeras, con cursos, simposios y talleres que abarcaron un programa muy ambicioso con temas de actualidad presentados por expertos y la posibilidad de interacción con los mismos. El COMMEC, generando lazos con otras sociedades, participó en la organización conjunta de simposios con la FEPIMCTI, la SCCM, la Neurocritical Care Society y por tercer año consecutivo en conjunto con la Asociación Mexicana de Terapia Intensiva Pediátrica, A.C.

El jueves llegó el día del quinto Tazón Alveolo 2019, concurso de ventilación mecánica donde participaron las sedes: Hospital Español, Hospital ABC, Hospital Juárez de México y Hospital de Especialidades No. 14 CMN «Adolfo Ruiz Cortines», del IMSS de Veracruz. El jurado estuvo integrado por profesores nacionales e internacionales de experiencia, quienes formularon preguntas en el formato ya conocido e imitado. El trofeo se disputó en la final entre el hospital Juárez y la sede campeona, el Hospital ABC de la Ciudad de México.
El día viernes se llevó a cabo la cena de clausura que fue amenizada por un grupo musical que nos hizo recordar, al ritmo de sus canciones, diferentes épocas, lo que nos motivó a reír y a compartir con amigos, maestros y compañeros unos minutos después de una semana de alto nivel académico.

Finalmente, posterior a la plenaria del sábado, el Dr. Jorge Sánchez Medina, actual presidente del COMMEC, dirigió unas palabras de agradecimiento a todos los asistentes, profesores e industria por su invaluable participación durante el congreso y declaró clausurado el XLVI Congreso Anual del COMMEC.

Esperamos contar con su presencia durante el próximo congreso que tendrá lugar en la ciudad de Mérida, Yucatán, del 9 al 14 de noviembre de 2020. Con su asistencia y participación será igualmente un gran acierto.

\footnotetext{
Correspondencia:

Dr. José de Jesús Zaragoza Galván Cincinnati 40 interior 201,

Col. Noche Buena, alcaldía Benito Juarez, CP 03810, Ciudad de México.

E-mail: zaragozagalvan@hotmail.com
} 